\title{
Basilar Artery Plaque Distribution, Pontine Infarction and Vertebrobasilar Artery Geometry: A Magnetic Resonance Imaging Study
}

\section{Jinmei Zheng}

Fujian Medical University Union Hospital

\section{Bin Sun}

Fujian Medical University Union Hospital

Ruolan Lin

Fujian Medical University Union Hospital

\section{Yongqi Teng}

Changle District Hospital of Fuzhou

\section{Enshuang Zheng}

Fujian Medical University Union Hospital

Xihai Zhao

Tsinghua University School of Medicine

\section{Yunjing Xue ( $\nabla$ xueyunjing@126.com )}

Fujian Medical University Union Hospital

\section{Research Article}

Keywords: Basilar artery atherosclerosis, plaque distribution, pontine infarction, vertebrobasilar artery, geometry

Posted Date: July 26th, 2021

DOl: https://doi.org/10.21203/rs.3.rs-640954/v1

License: (c) (1) This work is licensed under a Creative Commons Attribution 4.0 International License. Read Full License 


\section{Abstract}

Background: Basilar artery (BA) atherosclerosis is a common cause of posterior-circulation ischemic stroke. In this study, we investigate the relationship between BA plaque distribution, pontine infarction (PI) and vertebrobasilar artery (VBA) geometries.

Methods: 86 patients with BA plaque were enrolled in this study, the VBA geometry was classified into four configurations: walking, tuning fork, lambda, and no confluence. The AP-Mid-BA, Lateral-Mid-BA, and VA-BA angles were measured on three-dimensional time-of-flight magnetic resonance angiography. Patients underwent high-resolution magnetic resonance imaging to evaluate the BA plaque distribution (either anterior, posterior, or lateral wall). Acute and subacute PIs were identified by T2 weighted imagingfluid-attenuated inversion recovery and diffusion-weighted imaging.

Results: BA plaques in patients with PI were more frequently located at the posterior wall $(50.00 \%)$ than at the anterior $(10.00 \%)$ and lateral $(37.50 \%)$ walls $(P=0.028)$.

Compared with patients without pontine infarction, patients with pontine infarction were more likely to have plaque distributed at the posterior wall $(P=0.009)$. In the tuning fork group, BA plaques were evenly distributed. BA plaques were more frequently located at the lateral wall than at the anterior and posterior walls in patients with walking, lambda, and no confluence geometry (all $P \leq 0.05$ ). The AP-Mid-BA angle in patients with a tuning fork configuration $\left(14.95^{\circ} \pm 11.66^{\circ}\right)$ was lower than that in patients with other vascular geometries $(P=0.001)$; there was no significant difference in the VA-BA angle and lateral-mid-BA angle among the four VBA geometries $(P>0.05)$.

Conclusion: VBA configuration strongly influences BA plaque distribution and BA plaque distribution was associated with $\mathrm{PI}$.

\section{Background}

Posterior circulation stroke is defined by infarction occurring within the vascular territory supplied by the vertebrobasilar artery (VBA), and accounts for about $20-30 \%$ of all ischemic strokes [1, 2]. Klein et al. [3] used HR-MRI to observe 41 patients with pontine infarction $(\mathrm{PI})$, and found that more than $70 \%$ of patients had basilar artery (BA) plaque. BA atherosclerosis is a common cause of posterior circulation ischemic strokes, whose underlying mechanisms include artery-to-artery embolism, in situ thromboocclusion, and hemodynamic impairment. In addition, plaques located near the branch artery orifices may also induce pontine infarction[4]. Previous studies have demonstrated that coronary and middle cerebral artery atherosclerosis plaques tend to be distributed at positions opposite to the orifices of the perforating artery $[5,6]$. The BA forms the central core of the posterior circulation and is a rich source of perforating arteries; therefore, it is meaningful to investigate the relationship between BA plaque distribution and pontine infarction. 
Usually, the vertebrobasilar system is formed by the basilar and bilateral vertebral arteries. As the diameters of the left and right VAs and their anatomical course are different, a study by Yu J et al[7] classified the vertebrobasilar system into four geometric configurations: Walking, Tuning Fork, DominantLambda, and Hypoplasia-Lambda. Furthermore, they [7] demonstrated that the geometric configurations of the vertebrobasilar artery strongly influence BA plaque locations. Wake-buck et al. [8] reported a relationship between vertebrobasilar geometries and differences in hemodynamic distribution. In the study by Zheng J.M. et al [9], VBA geometry was qualitatively classified into four basic geometric configurations: Walking, Tuning Fork, Lambda, and No Confluence and found that the Walking, Lambda, and No Confuence geometry are associated with the presence of BA plaque. In this study, we want to investigate the relationship between BA plaque distribution, pontine infarction and vertebrobasilar artery geometries (Walking, Tuning Fork, Lambda, and No Confluence).

\section{Methods}

\section{Patients}

This study enrolled eighty-seven consecutive patients who presented with posterior circulation ischemic stroke to the Department of Neurology of our hospital from July 2017 to June 2018. Patients with the following characteristics were enrolled: (1) had BA plaque; and (2) displayed image quality sufficient for analysis. Patients were excluded if they had any of the following conditions: (1) non-atherosclerotic vasculopathy, such as dissection, arteritis, or Moya-Moya disease; (2) contraindications to MR imaging; (3) poor image quality due to motion artifact; or (4) their vascular geometry could not be classified. Based on these criteria, one patient, whose vascular geometry could not be classified, was excluded. Ultimately, 86 patients with BA plaque were included in our study.

This study was conducted following the Declaration of Helsinki and was approved by the Ethics Committee of Union Medical College Hospital Affiliated to Fujian Medical University. All patients signed an informed consent form to participate in the research.

\section{Image analysis}

Details of the high -resolution MRI protocol were described elsewhere [9]. On the axial CUBE images, if there was eccentric wall thickening, whereas the thinnest part was estimated to be $<50 \%$ of the thickest point by visual inspection, then, a plaque was identified [6]. All cross-sections with eccentric plaque were classified based on their plaque orientation being centered on the anterior, posterior, or lateral (left or right) sides of the vessel (Fig. 1) $[6,10]$. Each cross-section was grouped into one of four quadrants. In cases where the plaque was located at more than one quadrant, the quadrant with the maximal plaque thickness was chosen $[6,10]$, and we calculated the percentage of plaque distribution at the anterior, posterior, and lateral sides of the basilar wall. Acute and subacute pontine ischemic lesions were identified by T2WI-FLAIR and DWI. 
The diameter of the VA was measured using TOF-MRA. We measured three consecutive points, $3 \mathrm{~mm}$ apart, starting from the vertebrobasilar junction (both VAs and the BA), then, the diameter of each vessel was calculated as the average of the three measurements values $[7,11]$. The dominant vertebral artery was defined as the vertebral artery with the widest diameter (difference in diameter $\geq 0.3 \mathrm{~mm}$ )[7, 11]. Based on the TOF-MRA images, the vertebrobasilar artery geometry was group into four configurations: walking, tuning fork, lambda, and no confluence. The characteristics of each geometric configuration was defined elsewhere [9].

The AP-Mid-BA angle was measured using the anteroposterior (AP) view of 3D-reconstructed TOF-MRA, and the VA-BA angle and Lateral-Mid-BA angle were measured using the lateral view of the 3Dreconstructed TOF-MRA (Fig. 2). Imaginary lines were drawn from the mid-BA to the vertebrobasilar junction and the top of the BA in the AP and the lateral views, respectively (Fig. 2A and 2B)[12]. The maximum angles between these two imaginary lines were considered to be the AP-Mid-BA angle (AP view) and the Lateral-Mid-BA angle (lateral view). Using the same method, imaginary lines were drawn from the vertebrobasilar junction to the BA and the dominant VA; the angle between the two lines was considered the VA-BA angle (Fig. 2C)[12].

\section{Statistical analysis}

Statistical analysis was performed using IBM SPSS statistics software (version 19.0, IBM Corp., Armonk, NY, USA). The Shapiro-Wilk test of normality was used to investigate the distribution of data. Quantitative data are expressed as mean $\pm S D$, and qualitative data are expressed as percentages. Comparisons of $B A$ plaque distribution incidence in the anterior, posterior, and lateral sides of the BA wall were performed using Friedman's $M$ test. Comparisons of plaque distribution incidence between patients with and without pontine infarction were performed using the Mann-Whitney $\mathrm{U}$ test, and data comparisons of the four geometric configurations were performed by variance analysis or the Kruskal-Wallis test. $P<0.05$ was considered statistically significant.

\section{Results}

Of the 86 patients, there were 13 patients (median age, 67 years) with pontine infarction, and 73 patients without pontine infarction (median age, 73 years). Comparisons of clinical risk factors between patients with and without pontine infarction are summarized in Table 1. 
Table 1

Comparisons of Clinical Risk Factors between patients with $\mathrm{PI}$ and without $\mathrm{PI}$

\begin{tabular}{|c|c|c|c|}
\hline & $\mathrm{PI}(+)(\mathrm{N}=13)$ & $\mathrm{PI}(-)(\mathrm{N}=73)$ & $P$ \\
\hline Sex, male & $10(76.92)$ & $40(54.79)$ & $\# 0.222$ \\
\hline Age, y & $67(60.5-75.5)$ & 73(63.5-79.0) & $\& 0.136$ \\
\hline Smoking & $6(46.15)$ & 23(31.51) & *0.303 \\
\hline $\mathrm{BMI} \geq 28$ & $2(15.38)$ & $15(20.55)$ & $\# 1.000$ \\
\hline Hypertension & $11(84.62)$ & $58(79.45)$ & $\# 1.000$ \\
\hline Diabetes mellitus & $5(38.46)$ & $34(46.58)$ & $\star 0.588$ \\
\hline Hyperlipidemia & $7(53.85)$ & $31(42.47)$ & $\star 0.447$ \\
\hline $\mathrm{CHD}$ & $2(15.38)$ & $9(12.33)$ & $\# 0.670$ \\
\hline \multicolumn{4}{|c|}{ Values are presented as numbers (\%) or medians (interquartile ranges). } \\
\hline \multicolumn{4}{|c|}{ \#Comparisons were performed by Fisher's exact test; } \\
\hline \multicolumn{4}{|c|}{ *Comparisons were performed by chi-square test; } \\
\hline \multicolumn{4}{|c|}{ \&Comparison was performed by Mann-Whitney U test. } \\
\hline \multicolumn{4}{|c|}{$\mathrm{PI}$, pontine infarction; $\mathrm{BMI}$, body mass index; $\mathrm{CHD}$, coronary heart disease. } \\
\hline
\end{tabular}

\section{Correlation between BA plaque distribution and pontine infarction}

In 86 patients, plaques were identified in 716 slices. Only four patients had a one-slice plaque; most BA plaques involved multiple slices on high resolution magnetic resonance imaging (HR-MRI). The average length of $B A$ atherosclerosis plaque was $8.00 \mathrm{~mm}(4.50 \mathrm{~mm}-11.00 \mathrm{~mm})$ in 73 patients without pontine infarction and $9.00 \mathrm{~mm}(4.00 \mathrm{~mm}-11.50 \mathrm{~mm})$ in 13 patients with pontine infarction $(P=0.570)$.

BA plaques were more frequently located at the lateral walls than at the anterior and posterior walls in all 86 patients as well as in the 73 patients without pontine infarction. However, in the pontine infarction group, plaques were more frequently located at the posterior wall $(50.00 \%)$ than at the anterior $(10.00 \%)$ and lateral walls $(37.50 \%, P=0.028)$. Compared with patients without pontine infarction, patients with pontine infarction were more likely to have plaque distributed at the posterior wall $(P=0.009)$. The results of the basilar artery plaque distribution are shown in Table 2. 
Table 2

Basilar Artery Plaque Distribution

\begin{tabular}{|c|c|c|c|c|}
\hline & Anterior wall & Posterior wall & Lateral wall & P* \\
\hline $\begin{array}{l}\text { All patients }(\mathrm{N}= \\
86)\end{array}$ & $\begin{array}{l}19.09 \% \\
(0 \%-42.86 \%)\end{array}$ & $27.92 \%(0 \%-50.00 \%)$ & $\begin{array}{l}48.53 \% \\
(33.33 \%-66.67 \%)\end{array}$ & $<0.001^{1}$ \\
\hline $\mathrm{PI}(+)(\mathrm{N}=13)$ & $\begin{array}{l}10.00 \% \\
(0 \%-38.18 \%)\end{array}$ & $\begin{array}{l}50.00 \% \\
(23.64 \%-66.67 \%)\end{array}$ & $\begin{array}{l}37.50 \% \\
(31.67 \%-54.17 \%)\end{array}$ & $0.028^{2}$ \\
\hline $\mathrm{PI}(-)(\mathrm{N}=73)$ & $\begin{array}{l}20.00 \% \\
(0 \%-50.00 \%)\end{array}$ & $22.22 \%(0 \%-46.06 \%)$ & $\begin{array}{l}50.00 \% \\
(33.33 \%-66.67 \%)\end{array}$ & $<0.001^{3}$ \\
\hline$P^{\#}$ & 0.352 & 0.009 & 0.150 & \\
\hline \multicolumn{5}{|c|}{ Values are presented as medians (interquartile ranges). } \\
\hline \multicolumn{5}{|c|}{$\begin{array}{l}P^{\star} \text { indicates comparisons in the anterior, posterior, and lateral walls with Friedman's } \mathrm{M} \text { test; } P^{\#} \\
\text { indicates comparisons between patients with and without pontine infarction with the Mann-Whitney U } \\
\text { test; } \mathrm{Pl} \text {, pontine infarction. }\end{array}$} \\
\hline \multicolumn{5}{|c|}{${ }^{1}$ Anterior VS posterior, $P=0.910$; anterior VS lateral, $P<0.001$; posterior VS lateral, $P=0.002$} \\
\hline \multicolumn{5}{|c|}{${ }^{2}$ Anterior VS posterior, $P=0.056$; anterior VS lateral, $P=0.118$; posterior VS lateral, $P=1.000$} \\
\hline
\end{tabular}

\section{Correlation between BA plaque distribution and VBA geometry}

The percentage of plaque distribution at the anterior $(13.64 \%)$, posterior $(37.50 \%)$, and lateral walls $(37.50 \%, P=0.264)$ was similar in the 11 patients with Tuning Fork geometry. However, in the Walking, Lambda, and No Confluence groups, plaques were more frequently located at the lateral wall than at the anterior and posterior walls (all $P<0.05$, Table 3 ). 
Table 3

Vertebrobasilar Geometric Configuration and BA Plaque Distribution

\begin{tabular}{|c|c|c|c|c|}
\hline & Anterior wall & Posterior wall & Lateral wall & P* \\
\hline Walking (26) & $\begin{array}{l}15.34 \% \\
(0 \%-42.86 \%)\end{array}$ & $27.92 \%(0 \%-51.79 \%)$ & $\begin{array}{l}47.73 \% \\
(32.50 \%-66.67 \%)\end{array}$ & $0.019^{1}$ \\
\hline Tuning fork (11) & $\begin{array}{l}13.64 \% \\
(0 \%-50.00 \%)\end{array}$ & $37.50 \%(0 \%-66.67 \%)$ & $37.50 \%(0 \%-90.91 \%)$ & 0.264 \\
\hline Lambda (40) & $\begin{array}{l}25.00 \% \\
(0 \%-50.00 \%)\end{array}$ & $\begin{array}{l}24.35 \% \\
(2.94 \%-48.44 \%)\end{array}$ & $\begin{array}{l}48.53 \% \\
(34.38 \%-63.35 \%)\end{array}$ & $0.005^{2}$ \\
\hline $\begin{array}{l}\text { No confluence } \\
\text { (9) }\end{array}$ & $\begin{array}{l}11.11 \% \\
(0 \%-33.18 \%)\end{array}$ & $\begin{array}{l}30.00 \% \\
(12.42 \%-50.00 \%)\end{array}$ & $\begin{array}{l}50.00 \% \\
(36.51 \%-80.00 \%)\end{array}$ & $0.048^{3}$ \\
\hline$P^{\#}$ & 0.763 & 0.883 & 0.814 & \\
\hline \multicolumn{5}{|c|}{ Values are presented as medians (interquartile ranges). } \\
\hline \multicolumn{5}{|c|}{$\begin{array}{l}P^{*} \text { indicates comparisons in anterior, posterior, and lateral sides of the BA wall with the Friedman ' } M \\
\text { test; } P^{\#} \text { indicates comparisons in four groups with the Wallies } \mathrm{H} \text { test; BA, basilar artery. }\end{array}$} \\
\hline \multicolumn{5}{|c|}{${ }^{1}$ Anterior VS posterior, $P=1.000$; anterior VS lateral, $P=0.025$; posterior VS lateral, $P=0.249$} \\
\hline \multicolumn{5}{|c|}{${ }^{2}$ Anterior VS posterior, $P=1.000$; anterior VS lateral, $P=0.036$; posterior vs. lateral, $P=0.013$} \\
\hline${ }^{3}$ Anterior VS pc & $\mathrm{r}, \mathrm{P}=0.716$ & S lateral, $P=0.055$ & or vs. lateral, $\mathrm{P}=0.7$ & \\
\hline
\end{tabular}

The AP-Mid-BA angle in patients with the walking configuration $\left(36.13^{0} \pm 18.27^{0}\right)$, Lambda configuration $\left(24.84^{0} \pm 13.02^{0}\right)$, and No Confluence configuration $\left(24.17^{0} \pm 10.54^{0}\right)$ were higher than that in patients with Tuning Fork configuration $\left(14.95^{0} \pm 11.66^{\circ}\right)(P=0.001)$; however, there were no significant differences in the VA-BA and Lateral-Mid-BA angles among the four vertebrobasilar geometries $(P>0.05$, Table 4$)$. 
Table 4

Comparisons of angle in the four geometric configurations

\begin{tabular}{|c|c|c|c|c|c|}
\hline & $\begin{array}{l}\text { Walking } \\
(\mathrm{N}=26)\end{array}$ & $\begin{array}{l}\text { Tuning fork } \\
(\mathrm{N}=11)\end{array}$ & $\begin{array}{l}\text { Lambda } \\
(\mathrm{N}=40)\end{array}$ & $\begin{array}{l}\text { No confluence } \\
(\mathrm{N}=9)\end{array}$ & $P$ \\
\hline $\begin{array}{l}\text { AP-Mid-BA angle } \\
\left({ }^{0}\right)\end{array}$ & $36.13 \pm 18.27$ & $14.95 \pm 11.66$ & $24.84 \pm 13.02$ & $24.17 \pm 10.54$ & $\star 0.001^{1}$ \\
\hline VA-BA angle $\left({ }^{0}\right)$ & $\begin{array}{l}31.05 \text { (13.7- } \\
42.98)\end{array}$ & $\begin{array}{l}17.9(11.6- \\
28.6)\end{array}$ & $\begin{array}{l}24.7(18.4- \\
37.88)\end{array}$ & $\begin{array}{l}24.9(18.95- \\
33.4)\end{array}$ & \#0.362 \\
\hline $\begin{array}{l}\text { Lateral-Mid-BA } \\
\text { angle }\left(^{0}\right)\end{array}$ & $25.40 \pm 14.45$ & $22.35 \pm 10.3$ & $21.6 \pm 14.63$ & $23.26 \pm 8.5$ & *0.739 \\
\hline \multicolumn{6}{|c|}{ Values are presented as mean \pm standard deviation, or median (interquartile range). } \\
\hline \multicolumn{6}{|c|}{ *Comparisons were performed by variance analysis. } \\
\hline \multicolumn{6}{|c|}{ \#Comparison was performed by the Wallies $\mathrm{H}$ test. } \\
\hline \multicolumn{6}{|c|}{ AP, anteroposterior; VA, vertebral artery; BA, basilar artery. } \\
\hline \multicolumn{6}{|c|}{$\begin{array}{l}{ }^{1} \text { Walking vs. Tuning Fork, } P<0.001 ; \text { Walking vs. Lambda, } P=0.003 \text {; Walking vs. No Confluence, } P= \\
0.036 ; \text { Tuning Fork vs. Lambda, } P=0.048 ; \text { Tuning Fork vs. No Confluence, } P=0.160 ; \text { Lambda vs. No } \\
\text { Confluence, } P=0.900 \text {. }\end{array}$} \\
\hline
\end{tabular}

\section{Discussion}

In this study, it was observed that BA plaque in patients with pontine infarction was predominantly distributed in the posterior wall. Comparatively, BA plaques in patients without pontine infarction were more frequently distributed in the lateral wall $(50.00 \%)$ than in the anterior $(20.00 \%)$ and posterior $(22.22 \%)$ walls $(P \leq 0.001)$. Furthermore, we found that vertebrobasilar geometric configurations influence plaque distribution.

In a study by Yu et al. [10], BA plaques in the dorsal (posterior) and lateral walls were found to be associated with posterior ischemic stroke, where the branches of BA originate, and that BA plaques in asymptomatic patients were more likely located at the ventral (anterior) wall. In patients with pontine infarction, we found that BA plaque was predominantly distributed in the posterior wall (50.00\%); this finding is in line with previous study (9). However, in our study, the percentage of BA plaque distribution in the lateral wall $(50.00 \%)$ was higher than in the anterior $(20.00 \%)$ and posterior $(22.22 \%)$ walls in patients without pontine infarction. BA plaque in the inferior lateral wall or in the upper lateral wall all were defined as BA plaque of the lateral wall, however, as we all know, the penetrating artery of BA arises from the posterior and inferior lateral wall, thus, BA plaque in the inferior lateral wall more easily induced pontine infraction than in the upper lateral wall. In our study, BA plaque only in the upper lateral wall accounts for about $25 \%$ of BA plaque in the lateral wall, which may be explain the result that patients without pontine infarction have high incidence of BA plaque distribution in the lateral wall. 
A previous study[7] observed that geometric configurations strongly influence BA plaque distribution. In the present study, BA plaques were evenly distributed in the tuning fork geometry, which was consistent with the previous study (7). In the tuning fork geometry, the BA flow is roughly parallel and the velocity profile peak is rather central in the basilar artery, and the hemodynamic distribution is simple. However, in the vertebrobasilar arteries with walking, lambda, and no confluence configurations, BA plaques were more frequently occurred at the lateral wall, from where the penetrating artery arose (all $P \leq 0.05$ ). A study by Kim et al. [12] demonstrated that greater mid-BA angulation may enhance lateral plaque formation, and greater BA-VA angulation may enhance posterior plaque formation. In our research results, the APMid-BA angle in walking $\left(36.13^{0} \pm 18.27^{0}\right)$, Lambda $\left(24.84^{0} \pm 13.02^{0}\right)$, and No Confluence $\left(24.17^{0} \pm 10.54^{0}\right)$ geometries were greater than in the Tuning Fork geometry $\left(14.95^{0} \pm 11.66^{0}, P=0.001\right)$, and the greater APMid-BA in the Walking, Lambda, and No Confluence configurations may be induced the high percentage of BA plaque in the lateral wall among the three geometries. In addition, there were no significant differences in the VA-BA and Lateral-Mid-BA angles among the four vertebrobasilar geometries, which may be the reason that BA plaque distribution in the posterior wall among the four geometries have no significant differences.

Our study has many clinical implications. First, a previous study [10] reported that about two-thirds of BA plaques are located at the lateral and dorsal walls, where the penetrating artery arises, which have a high risk of pontine infarction, this suggests that BA plaque distribution may help us to assess the likelihood of ischemic stroke in the posterior circulation and reduce the risk of complications during stenting. We found about $50 \%$ of the total BA plaque located at the posterior wall where the penetrating artery arose in patients with pontine infarction, this is consistent with the previous study (9), which suggested that BA plaque in the posterior wall has a high risk of developing pontine infarction. Besides, we found that the incidence of BA plaque in the lateral wall was higher than in the anterior and posterior walls in patients without pontine infarction, and suggested that BA plaques in the upper lateral wall are unlikely to induce occlusion of the penetrating artery. Second, we demonstrated that geometric configurations and BA curvature angulation may influence the BA plaque distribution.

There are several limitations in this study. First, patients with vertebral artery atherosclerosis were not excluded from the study, thus, the hemodynamic effect of VA stenosis to BA atherosclerosis formation is unclear. Second, the sample size was small, only a single center was included. Finally, the hemodynamic distribution in the four geometries was not evaluated. Further studies measuring the hemodynamic distribution to elucidate the mechanism by which the geometry influences plaque distribution is necessary.

\section{Conclusions}

In conclusion, BA plaque in the posterior wall may be related to pontine infarction, besides, there are relationship between vertebrobasilar geometry and BA plaque distribution. Among the Walking, Lambda, and No Confluence geometries, BA plaques were often located at the lateral wall; while in the tuning fork geometry, the BA plaques were evenly distributed. 


\section{Abbreviations}

BA, basilar artery

VA, vertebral artery

VBA, vertebrobasilar artery

$\mathrm{PI}$, pontine infarction

3D-TOF-MRA, three-dimensional time-of-flight magnetic resonance angiography

HRMRI, high-resolution magnetic resonance imaging

\section{Declarations}

\section{Ethics approval and consent to participate}

The present study was approved by the Ethics Committee of Union Medical College Hospital Affiliated to Fujian Medical University. All methods were performed in accordance with the Declaration of Helsinki, and all patients provided their written informed consent.

\section{Consent for publication}

Not applicable.

\section{Availability of data and materials}

The datasets used and/or analyzed during the current study are available from the corresponding author on reasonable request.

\section{Competing interests}

The authors declare that they have no competing interests.

\section{Funding}

This work was supported by the Joint Funds for the Innovation of Science and Technology, Fujian Province [Grant number:2018Y9025].

\section{Authors' contribution}

Jinmei Zheng analyzed the data, and wrote the original draft. Jinmei Zheng, Bin Sun, Ruolan Lin, Yongqi Teng and Enshuang Zheng collected the data. Xihai Zhao analyzed the data, and edited the manuscript. Yunjing Xue supervised the whole study, and edited the manuscript. All authors read and approved the final manuscript. 


\section{Acknowledgments}

Not applicable.

\section{References}

1. Nouh A, Remke J, Ruland S. Ischemic posterior circulation stroke: a review of anatomy, clinical presentations, diagnosis, and current management. Front Neurol 2014;5:1-16.

2. Amin-Hanjani S, Pandey DK, Rose-Finnell L, et al. Effect of hemodynamics on stroke risk in symptomatic atherosclerotic vertebrobasilar occlusive disease. JAMA Neurol 2016;73:178-185.

3. Klein IF, Lavallée PC, Mazighi M, et al. Basilar artery atherosclerotic plaques in paramedian and lacunar pontine infarctions: a high-resolution MRI study. Stroke 2010;41:1405-409.

4. Bodle JD, Feldmann E, Swartz RH, et al. High-resolution magnetic resonance imaging: an emerging tool for evaluating intracranial arterial disease. Stroke 2013;44:287-292.

5. Watanabe $\mathrm{H}$, Yoshida $\mathrm{K}$, Akasaka $\mathrm{T}$, et al. Intravascular ultrasound assessment of plaque distribution in the ostium of the left anterior descending coronary artery. Am. J. Cardiol 1996;78:827-829.

6. Xu WH, Li ML, Gao S, et al. Plaque distribution of stenotic middle cerebral artery and its clinical relevance. Stroke 2011;42:2957-9.

7. Yu J, Zhang S, Li ML, et al. Relationship between the geometry patterns of vertebrobasilar artery and atherosclerosis. BMC Neurol 2018;18:83.

8. Wake-Buck AK, Gatenby JC, Gore JC. Hemodynamic characteristics of the vertebrobasilar system analyzed using MRI-based models. PLoS One 2012;7:e51346.

9. Zheng J, Sun B, Lin R, et al. Association between the vertebrobasilar artery geometry and basilar artery plaques determined by high-resolution magnetic resonance imaging. BMC Neurosci 2021; 22: 20.

10. Yu J, Li ML, Xu YY, et al. Plaque distribution of low-grade basilar artery atherosclerosis and its clinical relevance. BMC Neurol 2017;171:1-5.

11. Hong JM, Chung CS, Bang OY, et al. Vertebral artery dominance contributes to basilar artery curvature and peri-vertebrobasilar junctional infarcts. J Neurol Neurosurg Psychiatry 2009;80:10871092.

12. Kim BJ, Lee KM, Kim HY, et al. Basilar artery plaque and pontine infarction location and vascular geometry. J Stroke 2018;20:92-98.

\section{Figures}




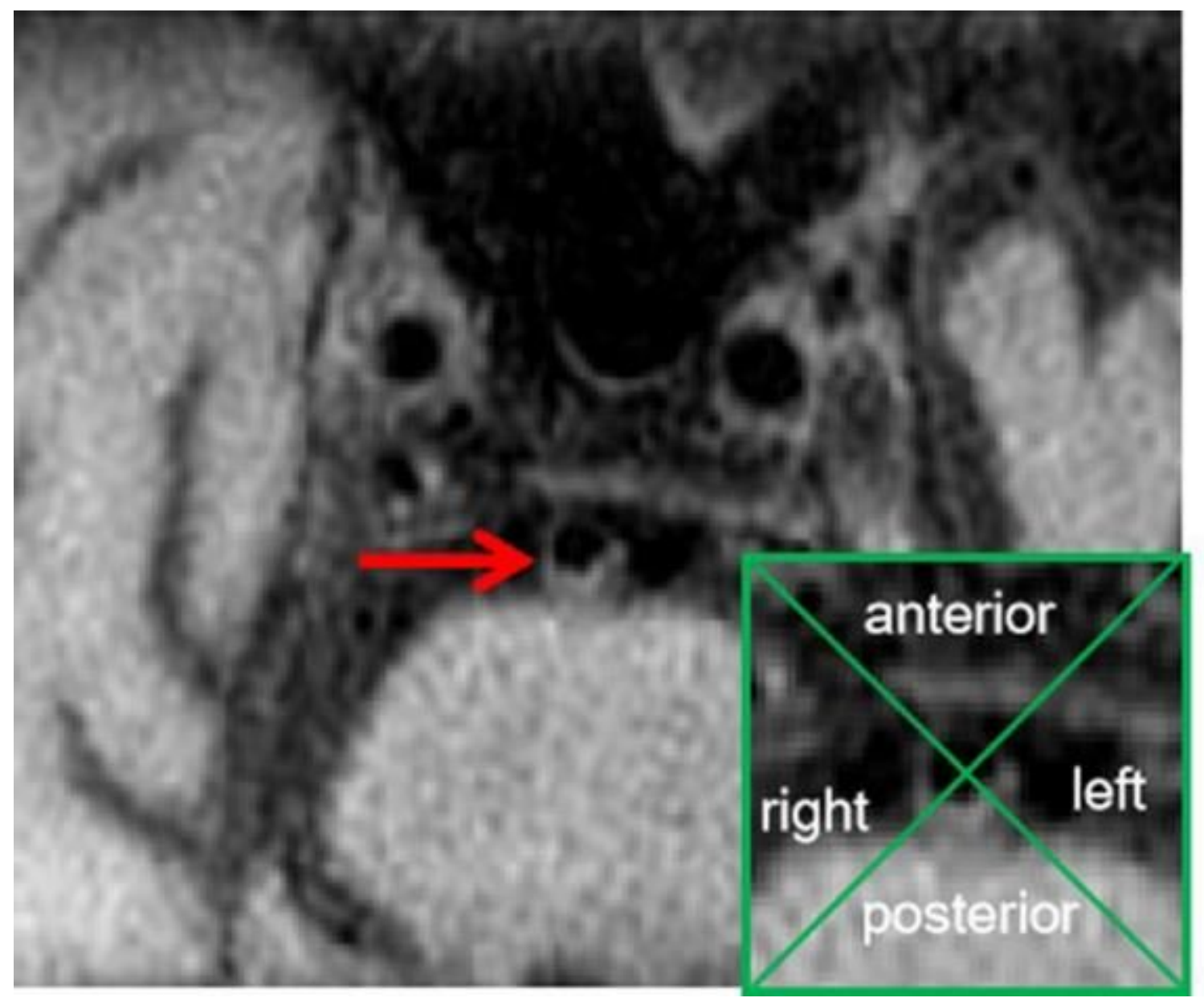

Figure 1

An alignment grid to demonstrate how each cross-section is divided into four quadrants.
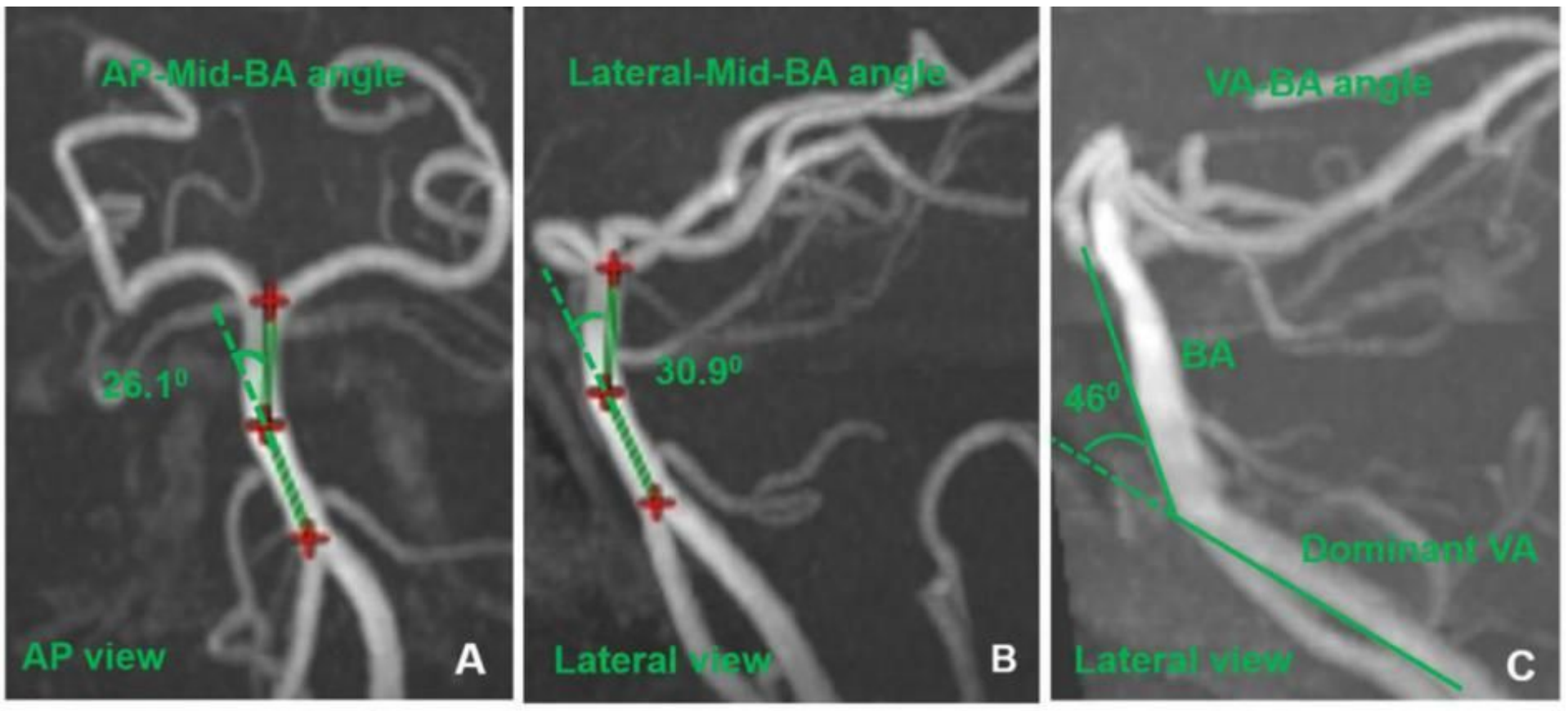

Figure 2 
The measurement of AP-Mid-BA angle (A), Lateral-Mid-BA angle (B) and VA-BA angle (C). BA: basilar artery; VA: vertebral artery. 